\title{
Study on the Mechanism of Renal Interstitial Fibrosis
}

\author{
Qi Jiang, Yingying Liu* \\ Department of Nephrology, China-Japan Union Hospital of Jilin University, Changchun, Jilin, 130033 \\ ${ }^{*}$ Corresponding author
}

Keywords: RIF, Mechanism, Medical Issue

\begin{abstract}
Interstitial fibrosis (RIF) refers to the pathological process of the kidney caused by a variety of damaging factors characterized by significant proliferation of fibroblasts within the interstitium and excessive deposition of extracellular matrix. RIF is the terminal stage of chronic kidney disease, the prognosis is poor; therefore, in-depth understanding of the formation mechanism of RIF to guide its prevention and treatment is of great significance. For a long time, epithelial-mesenchymal transition (EMT) is considered as an important cause of RIF. However, some scholars have put forward different opinions that EMT has nothing to do with renal interstitial fibrosis, Pericytes-mesenchymal transition and interstitial microvascular thinning are closely related.
\end{abstract}

\section{Introduction}

Ten years ago, Strutz et al. First reported the occurrence of EMT in renal fibrosis. In the rat model of anti-tubulointerstitial disease, the authors used FSP1 (fibroblast-specific protein-1) as a marker and found that renal tubular epithelial cells express FSP1, which is usually found only in fibroblasts Cells are expressed but epithelial cells are not expressed. So they put forward the hypothesis of EMT. Subsequently, Lan et. al. Also demonstrated on the morphological and cellular phenotypic characteristics the presence of EMT in residual kidneys after 5/6 nephrectomy. Consistent with animal experiments, EMT is also present in human renal biopsies. Rastaldi et al. [1] reported the renal biopsy results of 133 patients with different renal diseases. They found that the number of EMT-specific tubular epithelial cells (TECs) was closely related to the serum creatinine concentration and the degree of renal interstitial damage, indicating that EMT participation Human kidney fibrosis process. Now data show that in the event of kidney disease, most of the fibroblasts in the renal interstitium are transformed by the tubular epithelial cells derived from EMT. Selectively blocking EMT can significantly improve interstitial fibrosis in obstructive nephropathy in a murine model of the gene. Thus, EMT plays an important role in renal fibrosis.

\section{EMT main adjustment steps}

Recently, it has been found that EMT is a very complex process and is mainly regulated in the following four aspects at the cellular level: (1) loss of epithelial cell adhesion function; (2) expression of $\alpha$-smooth muscle actin ( $\alpha$-SMA) and muscle Kinetin recombination; tubule basement membrane destruction; improve cell migration and infiltration ability. Under normal circumstances, tubular epithelial cells through a variety of cell adhesion mechanisms closely linked to form a complete epithelial cell population. E-cadherin is a specific adhesion molecule receptor that plays a key role in maintaining the integrity and polarity of renal tubular epithelial cells. The first step in EMT is Snail, a zinc finger transcription factor that directly binds to the E-box at the start of E-cadherin, claudin and oc-culudin, thereby inhibiting their expression. $\beta$-catenin or Smad3 together activate lymphocyte augmenter and mediate Snail in lymphocyte nuclei. Presumably, the Snail transcription factor plays a key role in the early stages of EMT by inhibiting the gene expression of renal tubular epithelial cell adhesion molecules. The most commonly characterized tubular epithelial cells are marked by keratin; interstitial cells mainly express vimentin, which is a mesenchymal marker of cells; and $\alpha$-SMA is a marker protein of smooth muscle and myofibroblasts. Actin reorganization and $\alpha$-SMA expression not only marked epithelial morphological 
transformation, but also make it a migration, infiltration and contractile function. Similarly, actin cytoskeleton, cytoplasmic actin filaments also by epithelial keratin to mesenchymal transition of vimentin [2].

\section{EMT's main regulatory factors and cytokine interactions}

EMT by many growth factors, cytokines, hormones and extracellular matrix regulation, the specific signal transduction pathway is still not clear, which is currently one of the hot spots. Among many regulatory factors, TGF- $\beta 1$ is recognized as the most important pre-fibrotic factor. TFG- $\beta 1$ as a core factor, start and regulate the whole process of EMT. It is worth emphasizing that the process by which TGF- $\beta 1$ induces EMT is the main pathway by which TGF- $\beta 1$ leads to renal interstitial fibrosis under pathological conditions. TGF- $\beta$ induces phosphorylation of Smad 2 in tubular epithelial cells, which in turn leads to overexpression of Smad7, which inhibits TGF- $\beta$-mediated Smad2 activation, and inhibits EMT and collagen synthesis. So some scholars have proposed Smad signaling pathway can both promote and inhibit the EMT process. It has been demonstrated that the combination of epidermal growth factor (EGF) and TGF- $\beta$ can promote the transformation of tubule epithelial cells into mesenchymal cells. Like EGF, fibroblast growth factor-2 (FGF-2) decreases the expression of E-cadherin in TEC and induces the production of vimentin, FSP1 and $\alpha$-SMA, thereby promoting EMT, and this effect was significantly enhanced after the addition of TGF- $\beta 1$. These results suggest that these factors may all participate in EMT by regulating the expression of TGF- $\beta 1$. Experimental results show that TGF- $\beta 1$ and basic fibroblast growth factor-2 (bFGF-2) in the tubular epithelial cell line cultured in a positive feedback regulation. Interleukin-1 (IL-1) can also induce the phenotypic transformation of TEC, which is disappeared by the addition of anti-TGF- $\beta 1$ neutralizing antibody, indicating that the involvement of IL-1 in EMT is also mediated by TGF- $\beta 1$-induced. It has recently been reported that MMP- 2 is required for the induction of EMT and its effect is also considered to be related to the activity of TGF- $\beta 1$. Thus, TGF- $\beta 1$ is a central factor in the induction of EMT by various factors. TGF- $\beta$ can also induce the expression of TEC integrin-linked kinase (ILK) in a time-and dose-dependent manner and is regulated by the intracellular Smad signaling pathway. ILK is an intercellular serine / threonine protease that interacts with beta-integrin and many other cytoskeletal proteins in the cytoplasm and is involved in the regulation of integrin-mediated processes such as cell adhesion, Changes in cell morphology, deposition of extracellular matrix (ECM). Human proximal tubular cell (IL) secreted ILK can inhibit the expression of E-cadherin and synthesize fibronectin (FN) and MMP-2, thereby promoting cell migration and ECM deposition. In contrast, TGF- $\beta$-induced EMT was mostly absent in ILK-negative patients. The results of animal experiments suggest that administration of hepatocyte growth factor (HGF) can block the expression of ILK, inhibit the occurrence of EMT in TEC, and improve renal interstitial fibrosis. This shows that ILK also plays an important role in EMT [3].

On the other hand, there are some endogenous regulatory factors in the body can inhibit EMT. Hepatocyte growth factor (HGF) was originally found in rat sera partially excised from the liver because of its ability to stimulate the liver cells to synthesize DNA. HGF is a soluble cytokine, a glycoprotein of 728 amino acids. Its active form is a dimeric polypeptide consisting of a heavy chain ( $\alpha$ chain) and a light chain ( $\beta$ chain) linked by a disulfide bond. HGF can promote liver cell division, and mitogenic effect on a variety of epithelial cells, but also has a morphological role, is a strong mitogen, motor factors and morphogen. In the kidney, HGF is mainly expressed in renal interstitial cells, glomerular endothelial cells and macrophages. HGF acts specifically on the target cells, TEC, in the form of endocrine, autocrine and paracrine, and exerts its protective effect on the kidneys. Experiments by Yang et al. Confirmed that HGF can inhibit TEC transdifferentiation induced by TGF- $\beta 1$, but HGF can not reverse the collagen deposition and fibroblast proliferation that has been formed. Its anti-fibrotic effect is achieved by inhibiting ILK and thus blocking the EMT process. With further research, HGF may provide a new treatment for chronic kidney disease. 


\section{Renal interstitial fibrosis in the main effector cells function}

Because fibroblasts proliferate rapidly, producing large amounts of FN and collagen, particularly type I and type III collagen, has long been thought to play a key role in renal interstitial fibrosis. In fact, however, it remains unclear until now the exact relationship between the number of fibroblasts and the severity of interstitial fibrosis, since no specific markers have been found for fibroblasts in tissue. For the first time, Strutz et al. Cloned FSP1 and in the experimental fibrosis model, the number of cells expressing FSP1 was rapidly increased. Later, some people use the herpes simplex virus thymidase to control the expression of FSP1 and selectively remove the tissue fibroblasts to improve the fibrosis. This test for the first time directly proves that fibroblasts play an important role in the fibrosis of the body. Interstitial fibroblasts come from a wide range of sources. Kidney fibroblasts include: mesenchymal fibroblasts, circulating fibroblasts, fibroblasts transformed from epithelial cells, bone marrow-derived fibroblasts, and myofibroblasts expressing $\alpha$-SMA. Interstitial fibroblasts can synthesize ECM leading to renal interstitial fibrosis; hyperplasia can also occur cell phenotype changes to become myofibroblasts. Myofibroblasts produce large amounts of type I collagen and type III collagen expressing $\alpha$-SMA, which is a terminally differentiated cell between fibroblasts and smooth muscle cells and is a major source of extracellular matrix of renal interstitium. Myofibroblasts have been considered as activated fibroblasts. Recently, however, some scholars have found that the activated fibroblast cell adhesion molecule- 1 in the interstitium is positive but does not normally express $\alpha$-SMA, so it raises some questions about the original concept. Interstitial fibroblasts can be activated by certain cytokines such as TGF- $\beta$, PDGF and FGF-2. Studies have shown that patients with chronic kidney disease renal biopsy FGF-2 was significantly increased, and mainly exist in interstitial fibroblasts, and through anticrime stimulation of fibroblasts for injury repair. In vitro experiments show that FGF-2 is a strong mitogen of renal fibroblasts, but it does not stimulate type I collagen or FN synthesis. In addition, fibroblasts secrete various cytokines themselves, and these cytokines act on interstitial fibroblasts, resulting in the abnormal expression of certain genes that control their growth and apoptosis, leading to overgrowth and abnormal differentiation of fibroblasts Reduce death, increase the number of cells and collagen synthesis, eventually leading to renal interstitial fibrosis [4].

Macrophages can secrete a variety of factors involved in renal fibrosis, and in the stoma, the number of macrophage infiltrates correlates with the severity of fibrosis. Therefore, it has been thought that macrophages can promote renal fibrosis. This argument is supported by a number of subsequent studies. However, Nishida et al. unexpectedly found that exudative macrophages have an anti-fibrotic effect in renal fibrosis. After treatment of deadly bone marrow cells in irradiated wild type mice, they transplanted the kidneys from mice that lacked the AngII1 receptor (AT1R) gene into wild-type mice and received unilateral ureteral ligation simultaneously with untreated wild-type mice. Fourteen days later, the difference in renal lesions between the two groups of mice was observed [5]. The results showed that the number of infiltrating macrophages in the former decreased but the degree of renal fibrosis was heavier. Analysis found that the treatment group due to the absence of AT1R gene lead to monocytes and macrophages in peripheral blood infiltration and phagocytic function weakened, it can not clear excess ECM components and apoptotic cells, thereby increasing renal interstitial fibrosis. It has been demonstrated that urokinase cellular recep-tor (UPAR) participates in cell adhesion, invasion and angiogenesis. In the UP-AR - / - rats, the degree of renal fibrosis was more serious than that in the control group. The number of a-SMA-expressing cells and CD34 + endothelial cells was significantly increased, while the number of interstitial macrophages was significantly decreased. This study again demonstrates that macrophages do not exacerbate renal interstitial fibrosis and may even reduce fibrosis.

\section{Conclusions}

In the pathogenesis of renal interstitial fibrosis is complex and mutually influential, modern medicine although no specific drugs in the prevention and treatment of renal interstitial fibrosis, but has been found in many promising future breakthrough in prevention and treatment of renal 
interstitial fibrosis Such as TGF- $\beta$ antibodies, natural TGF- $\beta$ inhibitor decorin and the like, against the inhibition of TGF- $\beta$. CTGF is a novel fibroblast growth factor found in recent years. It has the effect of stimulating cell proliferation and ECM synthesis and is a downstream effector of TGF- $\beta$. It is the future research direction to study the receptor of CTGF and its signal transduction pathway one.

\section{References}

[1] Koesters R, Kaissling B, Lehir M, et al. Tubular overex-pression of transforming growth factor -beta1 induces au-tophagy and fibrosis but not mesenchymal transition of re-nal epithelial cells [J]. Am J Pathol, 2010, 177(2): 632 643.

[2] Iwano M, Plieth D, Danoff TM, et al. Evidence that fi-broblasts derive from epithelium during tissue fibrosis [J]. JClin Invest, 2002, 110(3): 341 350.

[3] Zeisberg M, Hanai J, Sugimoto H, et al. BMP-7 counter-acts TGF -beta1 -induced epithelial -to -mesenchymaltransition and reverses chronic renal injury [J]. Nat Med, 2003, 9(7): 964 968.

[4] Humphreys BD, Lin SL, Kobayashi A, et al. Fate tracingreveals the pericyte and not epithelial origin of myofibrob-lasts in kidney fibrosis [J]. Am J Pathol, 2010, 176(1): 85 97.

[5] Li L, Zepeda -Orozco D, Black R, et al. Autophagy is acomponent of epithelial cell fate in obstructive uropathy[J]. Am J Pathol, 2010, 176(4): 1767 1778. 